OPEN

SUBJECT AREAS:

PHYSICS

PLANETARY SCIENCE

Received

11 July 2014

Accepted

6 October 2014

Published

24 October 2014

Correspondence and requests for materials should be addressed to

J.C.R.-S. (cruiz@ cinvestav.mx)

\section{Penetration of Granular Projectiles into a Water Target}

\author{
Jorge González-Gutiérrez 1,2 , J. L. Carrillo-Estrada² \& J. C. Ruiz-Suárez'
} 1Centro de Investigación y de Estudios Avanzados, Unidad Monterrey, PIIT Autopista al Aeropuerto Km. 9.5, Apodaca, Nuevo León
66600, México, ${ }^{2}$ Instituto de Física, Benemérita Universidad Autónoma de Puebla, A. P. J-48, Puebla 72570, México.

The penetration of low-speed projectiles into a water target has been studied in the last several years to understand the physics behind the formation and collapse of cavities. In such studies, the projectiles employed were solid bodies or liquid drops. Here we report similar impact experiments using granular projectiles, with the aim to investigate how the morphology of the cavities is determined by the balance between the dynamic pressure exerted by the fluid and the cohesive strength of the impactors. From the results we present and discuss in this manuscript, we speculate on the dynamics of meteorite disintegration in the atmosphere of our planet.

ince the seminal work of Worthington and Cole more than one hundred years ago ${ }^{1}$, many scientists still aim to understand how the energy of a solid projectile, or the energy of a drop, is transferred into a liquid target $^{2-9}$. Not only the cavities they form are enthralling, but also their pinch-off and collapse, the jets they produce and the instability of the rims. Altogether, $\operatorname{low}^{4}$, moderate ${ }^{7}$, or high ${ }^{1,5}$ Reynolds numbers have been considered in such studies.

This article reports on experimental results on the same subject, but with a distinctive feature: the projectiles we used in our experiments are not solids but granular. We would like to find out if such projectiles either endure the impacts or not. We observe that they do when the cohesive energy that holds them together is larger than the impact energy, and do not otherwise. Depending on the outcome, the morphology of the water cavities and the dynamics of penetration is different. Our study aims to understand not only the reasons for such results but to conceive a possible geophysical implication.

\title{
Results
}

Three different impact events are shown in Fig. 1. Figure 1a shows the evolution of the cavity produced by a projectile with the highest mass $(m=42 \mathrm{~g})$ and an impact speed of $v_{i m p}=6.42 \mathrm{~m} / \mathrm{s}$, corresponding to a height h $=2.1 \mathrm{~m}$. The cavity is very similar to the one produced by a solid object. Figure $1 \mathrm{~b}$ depicts the cavity formed by the same projectile but this time for $v_{i m p}=8.97 \mathrm{~m} / \mathrm{s}(\mathrm{h}=4.1 \mathrm{~m})$. Clearly, in this more energetic impact grains suffer detachments due to lateral friction with the fluid. Finally, Fig. 1c shows the impact of a granular projectile with $m$ $=31.2 \mathrm{~g}$ and $v_{\text {imp }}=10.94 \mathrm{~m} / \mathrm{s}(\mathrm{h}=6.1 \mathrm{~m})$, where a greater cavity, due to the full pulverization of the projectile, is obtained. A video illustrating the sequence can be found in the supplementary material.

In Figs. 2a-d we show a sequence of the final cavities formed by different projectiles and impact velocities at a distance of penetration of three and a half projectile diameters, $z=3.5 \mathrm{D}$. It is clear that the process of erosion and/ or fragmentation changes the cavity morphologies. In Fig. 2e we depict the phase diagram of the cavity shapes in terms of the released height and packing fraction. We can clearly see that there are two regions: the dark and sky blue (triangles), corresponding to the case where the projectiles endure the impacts, and the yellow and red (dots and squares), where they fragment. This diagram prompt us to define a dimensionless number $\mathrm{C}_{c}$ as the ratio of two pressures: the comminution pressure $\mathrm{P}_{c}$ and the dynamic pressure $\mathrm{P}_{\mathrm{d}}=(1 / 2) \rho_{\mathrm{w}} v_{\text {imp }}{ }^{2}$, where $\rho_{\mathrm{w}}$ is the density of water. It is beyond the scope of this work to theoretically estimate the pressure needed to comminute the projectiles as a function of their cohesion properties. However, to circumvent this tough problem, we do measure the force needed to crush the projectiles by means of a simple technique: we compress them onto the plate of a balance. From the forces thus obtained, we estimate the comminution pressures, see Method section. When $\mathrm{C}_{\mathrm{c}} \approx$ 1 we obtain the black thick line in the phase diagram of Fig. 2e. Thus, when $\mathrm{C}_{\mathrm{c}}>1$ the cohesive forces dominate over the dynamic pressure and the aggregates don't fragment. Moreover, they produce cavities similar to those produced by solid projectiles (see Figs. $2 \mathrm{a}, \mathrm{b}$ ). When $\mathrm{C}_{\mathrm{c}}<1$ fragmentation takes place: the ball deforms during the impact, grains disperse and larger cavities form (Figs. $2 c, d$ ). 
a)
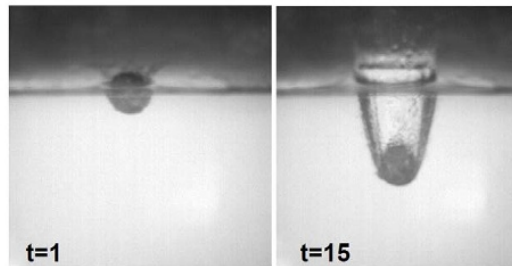

b)

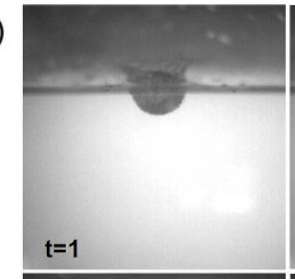

$t=15$

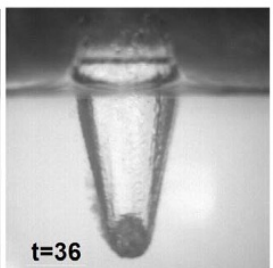

c)
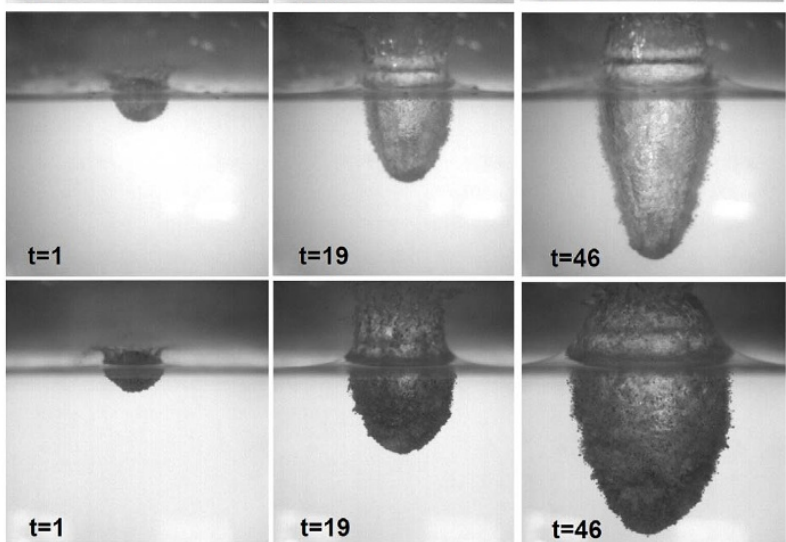

Figure $1 \mid$ A sequence of three cavity formation processes. (a) a granular projectile with $\eta=0.61(m=42 \mathrm{~g})$ released from $\mathrm{h}=2.1 \mathrm{~m}$ of height; (b) the same packing fraction but $\mathrm{h}=4.1 \mathrm{~m}$; (c) $\eta=0.45(m=32.2 \mathrm{~g})$ and $\mathrm{h}=6.1 \mathrm{~m}$. It is clear that full fragmentation produces greater cavities.

The dynamic equation that describes the penetration into the water medium after the projectile impacts with an initial velocity $v_{i m p}$ is: $m g-F_{b}-F_{d}=m a$, where $m$ is the mass of the projectile, $g$ gravity, $F_{b}$ the buoyant force, $F_{d}$ the drag force, and $a$ the acceleration. The depth of the projectile is equal to the cavity depth until the instant $t_{c}$ in which the cavity is closed. Therefore, for any instant $t<$ $t_{c}$, the depth of the projectile can be expressed as: $z(t)=-a_{0} t^{2} / 2+$ $v_{0} t$, where $a_{0}$ and $v_{0}$ are the deceleration and the initial velocity of immersion ${ }^{10}$.

In Fig. $2 \mathrm{f}$ we fit the experimental $z / \mathrm{D}$ vs $t$ data obtained for solid and granular projectiles of the same diameter $\mathrm{D}$ impacting the water target with a velocity $v_{i m p}=16.78 \mathrm{~m} / \mathrm{s}$. Clearly, there is no fragmentation at all for solid projectiles and the above equation describes the trajectory of these perfectly. In the case of granular projectiles released at low heights and high packing fractions, the fragmentation is small so they behave similarly as the solid ones (see the inset of Fig. 2f, where only the dynamics of the solid and the smallest packing fraction projectiles are shown). But when the impact force exceeds the cohesive forces of the granular projectiles (large heights and low packing fractions), fragmentation occurs, the balls deform, loose the coherence and the penetration dynamics is much slower. In this case, $z(t)$ strongly differs from the theoretical prediction, see Fig. $2 \mathrm{f}$.

Figure 3a shows the velocity of the cavity fronts (normalized with $v_{0}$ which is the velocity of the projectiles at a depth of $\mathrm{D} / 3$ ) as a function of depth (normalized with the diameter of the balls). These data were obtained by taking the derivatives of $z / \mathrm{D}$ vs $t$ of Fig. 2 f. Clearly, the lower the value of $\eta$ the larger the decrease of $v / v_{0}$ during immersion. Furthermore, Fig. $3 \mathrm{~b}$ shows the initial immersion velocity of the projectiles as a function of $1-\eta$ for different heights, see figure caption for details. When the height is small (squares), all the balls penetrate the fluid with the same $v_{0}$ regardless the value of $\eta$. Therefore, the drag force, which is proportional to $v_{0}$ is the same for small heights. However, $v_{0}$ decreases notably when they fragment (for small values of $\eta$ and larger heights), and this is why $a_{0}$ is smaller compared to the solid projectile, see inset of Fig. 3a. Note that for the largest height and $\eta, a_{0}$ is a)

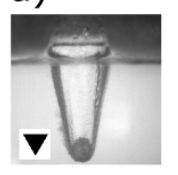

b)

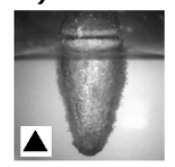

c)

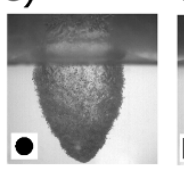

d)

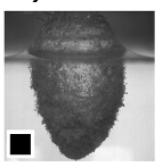

$\mathrm{P}_{\mathrm{c}}(\mathrm{kPa})$

e)

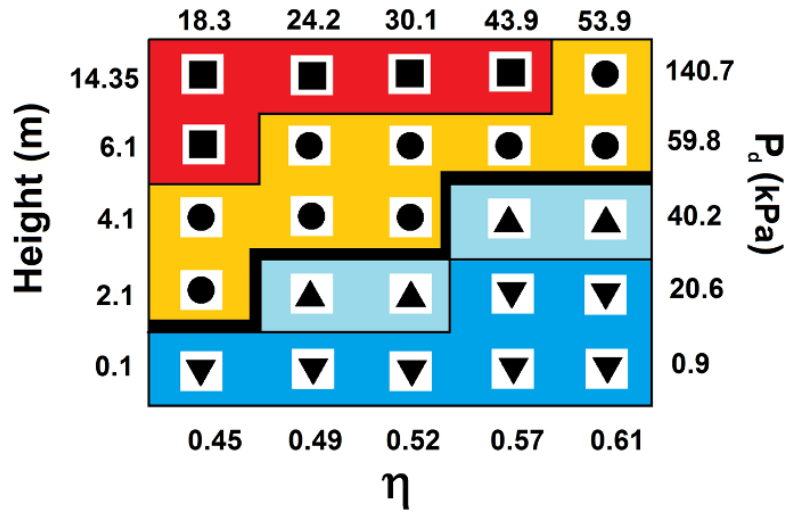

f)

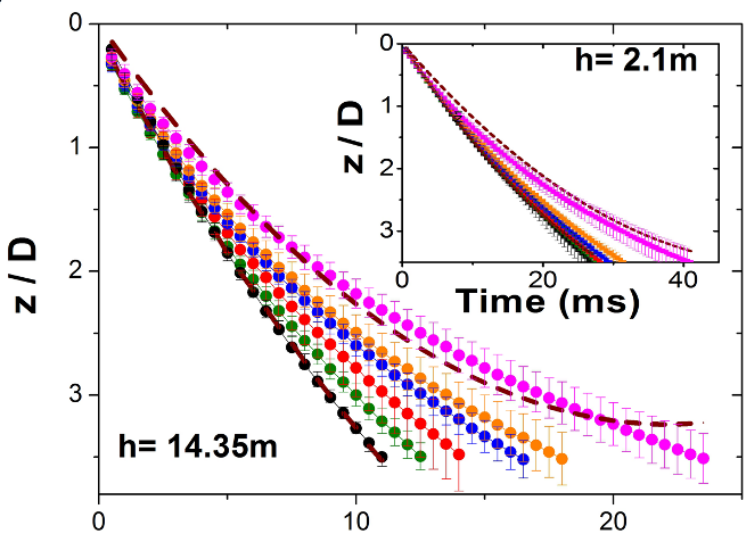

Time (ms)

Figure $2 \mid$ Phase diagram. (a) Cavity formed by a granular projectile after a penetration of three and a half diameters $(z=3.5 \mathrm{D})$. The released height was $h=2.1 \mathrm{~m}$ and $\eta=0.61$. The shape of the cavity is similar to the one produced by a solid projectile. (b)The cavity corresponds to granular projectile released at $\mathrm{h}=4.1 \mathrm{~m}$ for $\eta=0.61$. Here, particles detach and a slightly greater cavity is observed. (c) Almost full fragmentation occurs and the cavity widens $(\mathrm{h}=6.1 \mathrm{~m}$ and $\eta=0.55)$. (d) Full fragmentation takes place producing the largest cavity $(\mathrm{h}=6.1 \mathrm{~m}$ and $\eta=0.45)$. (e) Phase diagram $h-\eta\left(P_{c}-P_{d}\right)$, where the symbols correspond to the cavities shown in (a-d) (see text for details). (f) $z / \mathrm{D}$ vs $t$ for five packing fractions: 0.45 (pink), 0.49 (orange), 0.52 (blue), 0.57 (red), and 0.61 (green); plus the solid one (black), for $\mathrm{h}=14.35 \mathrm{~m}$. The dashed lines are the theoretically predicted trajectories, see text. The error bars were estimated using the standard deviation of five measurements for each event. Inset: the same as the main graph but $\mathrm{h}=2.1 \mathrm{~m}$, where it is clear that less fragmentation produces less spread on the curves.

maximum. A plausible explanation is that after the impact, the fragmentation of the granular projectile is not fully attained so the fragmented body sinks as a unit with an increased cross section. Therefore, the drag is greater. For lower packing fractions, the drag is exerted on the individual grains. In both cases, we follow the front of the cavity.

The volumes of the cavities are the only source of information to provide a quantitative measure of the fragmentation of the projec- 
a)
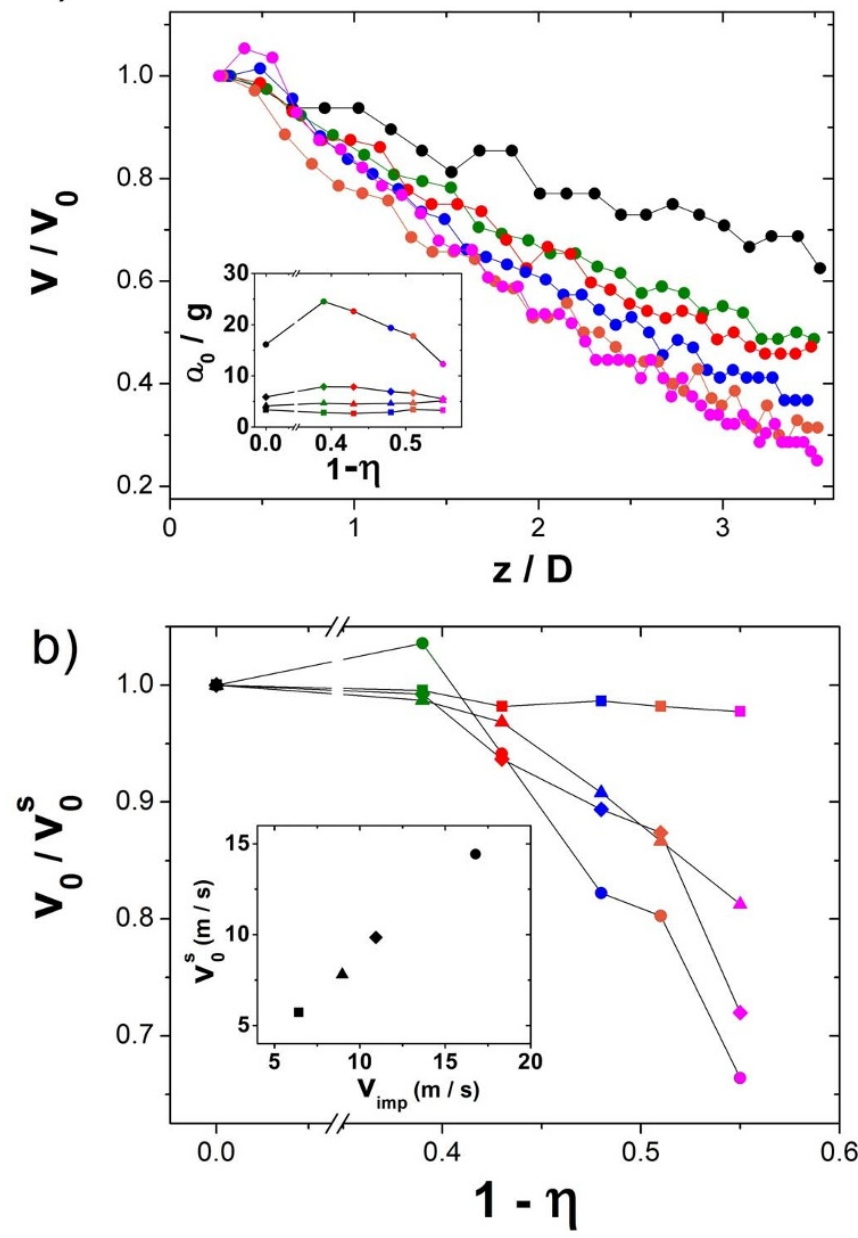

Figure 3 Dynamics of penetration. (a) $v / v_{0}$ vs $z / \mathrm{D}$ for a release hight of h $=14.35 \mathrm{~m}$ (dots) for different values of $\eta$ (see color nomenclature in the figure caption of Fig. 2). Inset: $a_{0}$ (normalised to $g$ ) as a function of $1-\eta$. (b) $v_{0} / v_{0}^{s}$ vs $1-\eta$ for different release heights: $2.1 \mathrm{~m}$ (squares), $4.1 \mathrm{~m}$ (triangles), $6.1 \mathrm{~m}$ (rhombus), and $14.35 \mathrm{~m}$ (dots). $v_{0}^{s}$ is the velocity of the solid balls measured at $z=D / 3$. Clearly, a substantial loss of impact energy is observed as $\eta$ decreases. The inset shows how $v_{0}$ changes with the impact velocity.

tiles. Since what we see with our fast camera is that the cavities have a parabolic shape, is then rather simple to determine the volume of the displaced fluid. The relation between the area of a parabola and the corresponding paraboloid of revolution is: $V=(3 / 16) \pi \lambda A$, where $\lambda$ and $A$ are the width and area of the parabola, respectively. In Fig. 4 we show $V$ (normalized to the volume of the projectile $V_{\text {imp }}$ ) as a function of $z$, for two projectiles with masses $m=31.2$ and $42 \mathrm{~g}$ having impact velocities between $v_{i m p}=6.42$ and $16.78 \mathrm{~m} / \mathrm{s}$. We include the volume of the cavities produced by solid impactors for equal masses and velocities, as a reference. Surprisingly, regardless the release height, namely, the impact velocity, the solid balls produce cavities with very similar volumes (black symbols), not only during the penetration but even at a depth of 3.5 D (see inset of Fig. 4). For high packings (green) and low impact velocities (squares), the cavities are of similar size than cavities made by solid balls. By increasing the impact velocity, the fragmentation of the granular projectiles takes place and the size of the cavity increases notably. Indeed, for loose projectiles (pink) fragmentation always occurs for the entire range of impact velocities (symbols) and the volume of the cavities is maximum.

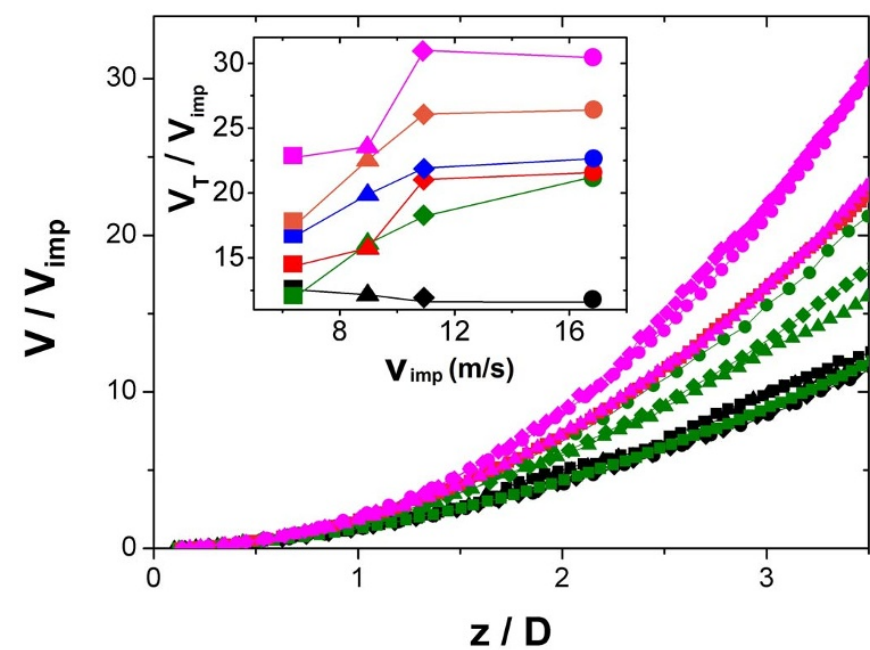

Figure $4 \mid$ Volume of the cavities produced by impactors. (a) $V / V_{i m p}$ vs z/D for granular and solid projectiles, see text for details. Inset: Total volumes, normalized also with the volume of the impactors, as a function of impact velocity for various packing fractions.

\section{Discussion}

Our results indicate that granular projectiles fully disintegrate when the dynamic force exerted by the fluid is larger than the cohesive force that holds them together. According to the phase diagram shown in Fig. 2e, $140 \mathrm{kPa}$ is a sufficient pressure for this to occur. Such phenomenon resembles the impact of water drops onto an immiscible liquid target, where fragmentation into a collection of non-coalescing daughter drops has been recently observed ${ }^{9}$. However, drops have surface tension and therefore non-coalescing daughter drops are rather few (it is required a very high impact energy to produce small drops in the millions ${ }^{11}$ ). In contrast, a relative small energy suffices to comminute our granular spheres into countless grains, see Method section.

Non-consolidated granular bodies were recently used by us to perform impact cratering experiments on sand, hopefully to learn about geophysical phenomena where graininess is a relevant issue $^{12-14}$. Here, based on the results presented in the previous section, we aim to learn about the entrance of a meteor in the atmosphere of our planet considering the above disintegration results.

Since the high-energy entries of large granular meteors into Earth's atmosphere are rare events, and therefore we cannot witness their endurance or destruction, we carried out low-energy impacts into another type of "atmosphere": water. Such "atmosphere" is much denser than air. The idea is to compensate the low laboratory energies using, as a target, a medium whose density is six orders of magnitude higher than the density of air at high altitudes. In this way, the low value of $v_{i m p}{ }^{2}$ (in the expression of the dynamic pressure) is compensated by the high value of $\rho$ (with the result of reaching the same dynamic pressure existing in a geophysical impact). Moreover, the water tank is like a "bubble chamber" where we can spy the impact events.

We display two important numbers: the Reynolds (Re) and the comminution number $\mathrm{C}_{\mathrm{c}}$ we defined above. For the impact velocity of $16.78 \mathrm{~m} / \mathrm{s}, \mathrm{Re}=612470$ (bear in mind that the diameter of the spheres is $3.65 \mathrm{~cm}$ and the kinematic viscosity of water is $1 \times$ $10^{-6} \mathrm{~m}^{2} / \mathrm{s}$ ) and $\mathrm{C}_{\mathrm{c}}$ according to the phase diagram of Fig. 2, is approximately 0.13 .

The same value of Re emerges if we consider a projectile ten times as large ( $30.74 \mathrm{~cm}$ of diameter), falling in the atmosphere of the Earth with a velocity of $17000 \mathrm{~m} / \mathrm{s}$ at an altitude of $45 \mathrm{~km}$ (where the kinematic viscosity up there is ${ }^{15}: 0.008533 \mathrm{~m}^{2} / \mathrm{s}$ ). Furthermore, in our experiments, at the largest speed, $\mathrm{P}_{\mathrm{d}}$ is $141 \mathrm{KPa}$. Enough, as we 


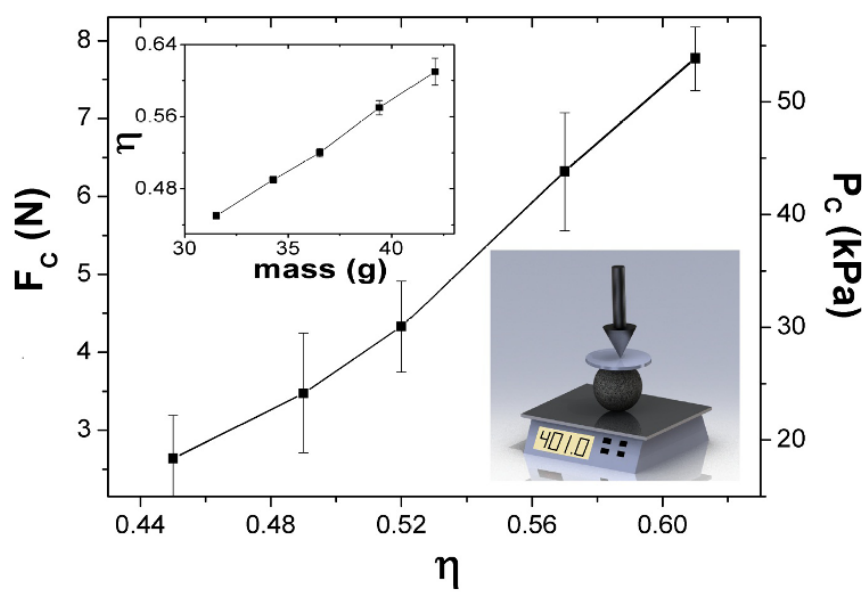

Figure $5 \mid$ Comminution pressures and packings. Forces applied to crush the granular balls (see lower inset), as a function of packing fraction. Dividing these forces by the dynamic pressures, a contact area of $1.44 \mathrm{~cm}^{2}$ (a circle of $0.677 \mathrm{~cm}$ of radius) is found. The area is large because the sphere crumbles a bit to the touch when the upper and bottom plates make contact. With such area we estimate the values of $\mathrm{P}_{\mathrm{c}}$ (right axis). Upper inset: linear relation between packing fraction and mass.

see in Fig. 2, to utterly crush even the most compact projectiles. Since air at $45 \mathrm{~km}$ of altitude has a density of $0.001965 \mathrm{Kg} / \mathrm{m}^{3}$, the $30.74 \mathrm{~cm}$ ball made of the same material and same packing fraction will be subjected, in our thought experiment, to a pressure of $284 \mathrm{KPa}$. Therefore, same Reynolds and half Cc imply that the projectile would disintegrate twice as easily.

Consider now a $500 \mathrm{~m}$ granular meteor with the same entrance velocity $(17 \mathrm{~km} / \mathrm{s})$. This hypothesis (that meteors have a granular nature) is not a strong supposition, since mounting evidence shows that asteroids are indeed granular agglomerates ${ }^{16-21}$. The Reynolds in this case would be $1 \times 10^{9}$ and $\mathrm{P}_{\mathrm{d}}$ the same $284 \mathrm{KPa}$ than before (at $45 \mathrm{~km}$ of height). However, $\mathrm{C}_{\mathrm{c}}$ would be much less than one, since the only way to hold asteroids of this kind together is with mild van der Waals forces according to D. J. Scheeres et $\mathrm{al}^{21}$ (asteroid, of course, were not made billions of years ago by pressing grains in a laboratory as we do). Therefore, since $\mathrm{C}_{\mathrm{c}}$ is much less than one and the very high Re acts in favour, the $500 \mathrm{~m}$ granular body would start to disintegrate completely at $45 \mathrm{~km}$ of altitude.

Overall, we report impact experiments of granular projectiles into a water target. Our goal was to investigate the dynamics of these impacts and construct a phase diagram to classify the form of the cavities, as a function of the impact energy and packing fraction of the projectiles. Using such diagram, we were able to learn about the fate of such impactors. We believe that our results may give a clue to understand why our planet has so few craters compared, for example, with the Moon. Were they comminuted by Earth's atmosphere at around $45 \mathrm{~km}$ of height?

\section{Methods}

The projectiles are prepared in the following way: $360 \mathrm{~g}$ of sand $\left(\rho=2.72 \mathrm{~g} / \mathrm{cm}^{3}\right)$ and $25 \mathrm{ml}$ of water are thoroughly mixed until a homogeneous mud is obtained. Approximately $35 \mathrm{ml}$ of this material is used to overfill two metallic spherical shells. Once they are pressed one against the other, a consolidated (yet fragile) ball with diameter $\mathrm{D}=3.65 \pm 0.3 \mathrm{~cm}$ is formed. By changing the mass of the wet sand used and the pressure exerted, balls with the same diameter and five different packing fractions $\eta$ are obtained. The aggregates are dried in an oven at $160^{\circ} \mathrm{C}$ for 2 hours and after that, weighed on an analytical balance. The dried balls can be grabbed with the fingers as if they were solid balls, although some care is needed to keep their integrity. The projectiles were classified in five groups as a function of mass: $m=31.2,34.9$, $36.6,39.3$, and $42 \pm 0.5 \mathrm{~g}$, corresponding to packing fractions $\eta=0.45,0.49,0.52$, 0.57 , and $0.61 \pm 0.01$, respectively. The force needed to crush the balls, the corresponding pressures and the correlation between $\eta$ and $m$ are depicted in Fig. 5 .
120 litres of water were poured into a Plexiglas tank with dimensions $60 \times 50 \times$ $60 \mathrm{~cm}$. An experiment starts when a granular projectile is released from a height $\mathrm{h}$, measured from the water surface, into this tank. After the impact, the projectile produces a cavity that is filmed at $2000 \mathrm{fps}$ with a high speed camera (DRS Lightning RDT Plus). $\mathrm{h}$ is varied from 0.1 to $14.30 \mathrm{~m}$, corresponding to impact velocities from 1.4 to $16.78 \mathrm{~m} / \mathrm{s}$. The granular projectiles produce well-defined cavities whose shape depends on the packing fraction. We observe either no fragmentation at all, minor erosion, coarse fragmentation, and full pulverization of the projectiles. $120 \mathrm{ml}$ of polyvinyl aluminum and $20 \mathrm{~g}$ of an anionic polymer, that together promote flocculation, are used to ensure that the fluid clears out in less than five minutes when the projectiles disintegrate and disperse.

1. Worthington, A. M. \& Cole, R. S. Impact with a liquid surface, studied by the aid of instantaneous photography. Philos. Trans. R. Soc. London, Ser. A 194, 175 (1900).

2. Cheny, J.-M. \& Walters, K. Rheological influences on the splashing experiment. J. Non-Newtonian Fluid Mech. 86, 185 (1999).

3. Yarin, A. L. Annu. Drop impact dynamics: Slashing, Spreading, Receding, Bouncing. Rev. Fluid Mech. 38, 159 (2006).

4. Lee, M., Longoria, R. G. \& Wilson, D. E. Cavity dynamics in high-speed water entry. Phys. Fluids 9, 540 (1997).

5. Duclaux, V., Caillé, F., Duez, C., Ybert, C., Bocquet, L. \& Clanet, C. Dynamics of transient cavities. J. Fluid Mech. 591, 1 (2007).

6. Duez, C., Ybert, C., Clanet, C. \& Bocquet, L. Making a splash with water repellency. Nat. Physics 3, 180-183 (2007).

7. Le Goff, A., Quéré, D. \& Clanet, Ch. Viscous cavities. Phys. Fluids 25, 043101 (2013).

8. Zhang, L. V., Brunet, P., Eggers, J. \& Deegan, R. D. Wavelength selection in the crown splash. Phys. Fluids 22, 122105 (2010).

9. Lhuissier, H., Sun, C., Prosperetti, A. \& Lohse, D. Drop Fragmentation at Impact onto a Bath of an Immiscible Liquid. Phys. Rev. Lett. 110, 264503 (2013).

10. Aristoff, J. M., Truscott, T. T., Techet, A. H. \& Bush, J. W. M. The water entry of decelerating spheres. Phys. Fluids 22, 032102 (2010).

11. Cohen, R. D. Shattering of a liquid drop due to impact. Proc. R. Soc. Lond. A 435, 483 (1991).

12. Pacheco-Vazquez, F. \& Ruiz-Suarez, J. C. Impact craters in granular media: grains against grains. Phys. Rev. Lett. 107, 218001 (2011).

13. Bartali, R., Rodriguez-Liñan, G. M., Nahmad-Molinari, Y., Sarocchi, D. \& RuizSuárez, J. C. Role of the granular nature of meteoritic projectiles in impact crater morphogenesis. arXiv:1302.0259v2, (2013).

14. Ruiz-Suárez, J. C. Penetration of projectiles into granular targets. Rep. Prog. Phys. 76, 066601 (2013).

15. Glenn Research Center, NASA. The similarity parameter calculator (2006). Available: http://www.grc.nasa.gov/WWW/BGH/airsim.html (Date of access: 03/ 15/2014).

16. Yeomans, D. K. et al. Estimating the mass of asteroid 253 Mathilde from tracking data during the NEAR flyby. Science 278, 2106 (1997).

17. Veverka, J. et al. NEAR's flyby of 253 Mathilde: images of a C asteroid. Science 278, 2109 (1997).

18. Love, S. G., Horz, F. \& Brownlee, D. E. Target porosity effects in impact cratering and collisional disruption. Icarus 105, 216-24 (1993).

19. Richardson, D. C., Leinhardt, Z. M., Melosh, H. J., Bottke, W. F. Jr. \& Asphaug, E. Gravitational Aggregates: Evidence and Evolution, in Asteroid III, ed Bottke, W. F. Jr., Cellino, A., Paolicchi, P. \& Binzel, R. P. (The University of Arizona Press, Tucson) 501-15 (2002).

20. Housen, K. R. \& Holsapple, K. A. Impact cratering on porous asteroids. Icarus 163, 102-119 (2003)

21. Scheeres, D. J., Hartzell, C. M., Sánchez, P. \& Swift, M. Scaling forces to asteroid surfaces: The role of cohesion. Icarus 210, 968-84 (2010)

\section{Acknowledgments}

This work has been supported by Conacyt, Mexico, under Grants 101384 and 104616. J. G. G. acknowledges a fellowship from CONACyT, Mexico. Useful discussions with Y. Nahmad, R. Bartali and R. Godoy are acknowledged. We acknowledge the CIIDIT-UANL for allowing us to perform the experiments in their emergency stairs.

\section{Author contributions}

J.G.G. carried out all the experiments and performed the analysis of the data. J.G.G. prepared all figures. J.G.G., J.L.C.E. and J.C.R.S. contributed in the discussion of the results and preparation of the manuscript. J.C.R.S. supervised the research.

\section{Additional information}

Supplementary information accompanies this paper at http://www.nature.com/ scientificreports

Competing financial interests: The authors declare no competing financial interests. How to cite this article: González-Gutiérrez, J., Estrada, J.L.C.- \& Suárez, J.C.R.Penetration of Granular Projectiles into a Water Target. Sci. Rep. 4, 6762; DOI:10.1038/ srep06762 (2014) 

NoDerivs 4.0 International License. The images or other third party material in this article are included in the article's Creative Commons license, unless indicated otherwise in the credit line; if the material is not included under the Creative
Commons license, users will need to obtain permission from the license holder in order to reproduce the material. To view a copy of this license, visit http:// creativecommons.org/licenses/by-nc-nd/4.0/ 